\title{
TI.77.1
}

\section{Reconnections: Managing Academic Networks With New Requirements}

- PDF: Internet2_Reconnections_Workshop.pdf

- Text: Internet2_Reconnections_Workshop.txt

\section{More Information}

\begin{tabular}{|l|l|}
\hline Repository ID & TI.77.1 \\
\hline Persistent URL & http://doi.org/10.26869/TI.77.1 \\
\hline Title & Reconnections: Managing Academic Networks With New Requirements \\
\hline Authors & Ken Klingenstein \\
\hline Sponsor & SALSA \\
\hline Review & \\
\hline Status & Legacy \\
\hline Publish Date & September 7,2005 \\
\hline DOI & $10.26869 /$ TI.77.1 \\
\hline Signature & \\
\hline Deprecated & No \\
\hline Future Review & \\
\hline Supersedes & \\
\hline Format & PDF, Text \\
\hline Related Docs & \\
\hline Development Location & \\
\hline IP Framework & \\
\hline Subject Tags & middlewarerescue \\
\hline Notes & \\
\hline & \\
\hline
\end{tabular}

